\title{
Quality and turnaround times of viral load monitoring under prevention of mother-to-child transmission of HIV Option B+ in six South African districts with a high antenatal HIV burden
}

\author{
N K Ngandu, ${ }^{1 \star} \mathrm{PhD} ;$ D F Nsibande, ${ }^{1 *} \mathrm{MPH} ;$ V Magasana, ${ }^{1 \star} \mathrm{MPH} ; \mathrm{W}$ Chirinda, ${ }^{1} \mathrm{PhD} ; \mathrm{T}$ Mbira, ${ }^{1 *} \mathrm{BSc}$ Hons; G G Sherman, ${ }^{2,3} \mathrm{MD}, \mathrm{PhD}$ \\ A E Goga, ${ }^{1 *, 4} \mathrm{MD}, \mathrm{PhD}$ \\ ${ }^{1}$ Health Systems Research Unit, South African Medical Research Council, Cape Town, South Africa \\ ${ }^{2}$ Department of Paediatrics and Child Health, Faculty of Health Sciences, University of the Witwatersrand; and Centre for HIV and STIs, \\ National Institute for Communicable Diseases, Johannesburg, South Africa \\ ${ }^{3}$ National Institute for Communicable Diseases, National Health Laboratory Service, Johannesburg, South Africa \\ ${ }^{4}$ Department of Paediatrics, School of Medicine, Faculty of Health Sciences, University of Pretoria, South Africa \\ * Current affiliation: HIV Prevention Research Unit, South African Medical Research Council, Cape Town, South Africa
}

Corresponding author: N K Ngandu (nobubelo.ngandu@mrc.ac.za)

\begin{abstract}
Background. Barriers to monitoring maternal HIV viral load (VL) and achieving $90 \%$ viral suppression during pregnancy and breastfeeding still need to be understood in South Africa (SA).

Objectives. To measure quality of VL care and turnaround times (TATs) for returning VL results to women enrolled in the prevention of mother-to-child transmission of HIV (PMTCT) programme in primary healthcare facilities.

Methods. Data were obtained from a 2018 cross-sectional evaluation of the PMTCT Option B+ programme in six SA districts with high antenatal and infant HIV prevalence. Quality of VL care was measured as the proportion of clients reporting that results were explained to them. TATs for VL results were calculated using dates abstracted from four to five randomly selected facility-based client records to report overall facility 'short TAT' ( $\geq 80 \%$ of records with TAT $\leq 7$ days). Logistical regression and logit-based risk difference statistics were used. Results. Achieving overall short TAT was uncommon. Only 50\% of facilities in one rural district, zero in one urban metro district and $9-38 \%$ in other districts had short TAT. The significant difference between districts was influenced by the duration of keeping results in facilities after receipt from the laboratory. Expected quality of VL care received ranged between $66 \%$ and $85 \%$. Client-related factors significantly associated with low quality of care, observed in two urban districts and one rural district, included lower education, recent initiation of antiretroviral treatment and experiencing barriers to clinic visits. Experiencing clinic visit barriers was also negatively associated with short TATs.

Conclusions. We demonstrate above-average quality of care and delayed return of results to PMTCT clients. Context-specific interventions are needed to shorten TATs.
\end{abstract}

S Afr Med J 2021;111(8):759-767. https://doi.org/10.7196/SAMJ.2021.v111i8.15496

Optimal monitoring of the HIV viral load (VL) in antenatal and postnatal clients is key to identifying those at risk of mother-to-child transmission of HIV (MTCT). ${ }^{[1]}$ Timeous identification of clients who are failing treatment will facilitate adherence and treatment interventions and lower MTCT risk. Globally, HIV elimination is aimed to be achieved by 2030, and one way to ensure good progress is to meet the HIV $90-90-90$ targets at national level, ${ }^{[2]}$ i.e. $90 \%$ of people living with HIV should know their status, $90 \%$ of people living with HIV should be on antiretroviral therapy (ART), and 90\% of those on ART should be virally suppressed. ${ }^{[2]}$ In South Africa (SA), the prevention of MTCT (PMTCT) antenatal care cascade manages to achieve $>90 \%$ coverage for HIV testing and for ART initiation. ${ }^{[3]}$ The service delivery performance of these two PMTCT indicators is further strengthened by periodic monitoring and evaluation through the national health information systems and the antenatal sentinel surveys. ${ }^{[3]}$ Systematic tracking of the third 90 for the PMTCT programme in SA was only adopted in 2019. ${ }^{[4]}$

During the period 2015 - 2019, the SA PMTCT guidelines for monitoring VL recommended 6-monthly VL testing for mothers who were virally suppressed $(\leq 1000$ copies $/ \mathrm{mL}$ ) and monthly testing for unsuppressed mothers. ${ }^{[5]}$ The turnaround times (TATs) from blood draw to returning results to clients should not delay clinical management. Although the 2015 guidelines expected results to be received by clients within 2 weeks and the recent 2019 guideline revisions require VL-informed care to be issued within 1 week, it is unclear what TATs are currently achieved in the public primary healthcare setting. ${ }^{[4,5]}$ The quality of VL care service is also important, as it has the potential to influence client participation and TATs. Quality of care, in this case, would include ensuring that all blood samples yield a VL result and all VL-tested mothers receive their results in the shortest time possible. In addition, the meaning and implication of the mother's VL result need to be explained to her during collection of results.

\section{Objectives}

Considering the scarcity of information around the third 90 for antenatal and postnatal clients, we sought to understand two aspects of routine VL monitoring in high antenatal HIV prevalence districts 
of SA. Firstly, we sought to document average VL TATs achieved by facilities, and secondly, we implemented a simple measure of selfreported quality of client care during receipt of VL results. We used client recall of the healthcare provider explaining the meaning of the VL results as a measure of quality, as this is critical for improving the client's attitude towards healthcare uptake. Lastly, using multivariable analyses, we assessed facility structural factors associated with TAT, client-related factors associated with the self-reported quality of care, and the relationship between average TAT and VL quality of care at facility level.

\section{Methods \\ Study design}

A cross-sectional process evaluation of PMTCT Option B+ implementation was conducted in six SA districts between February and June 2018, to assess programme performance 2 years after national roll-out. Study districts were selected from 25 districts with the highest infant HIV incidence and antenatal HIV prevalence in 2014 - 2015, and were: (i) a periurban district, OR Tambo in Eastern Cape Province (Periurban EC); (ii) two urban metros, Ekurhuleni in Gauteng Province (Urban GP) and eThekwini in KwaZulu-Natal Province (Urban KZN); (iii) two rural districts, Greater Sekhukhune in Limpopo Province (Rural LP) and Bojanala in North West Province (Rural NW); and (iv) one periurban district with a large rural setting, Ehlanzeni in Mpumalanga Province (Rural/periurban MP). The process evaluation was carried out at managerial/policymaker level (interviews), service delivery level (interviews with healthcare workers and assessment of facility registers/documentations), and user level (interviews with mothers). Data from facility registers and maternal interviews were used for this analysis.

Sample sizes were determined to estimate district-level maternal data for key PMTCT indicators, using an absolute precision of $10 \%$ and a design effect of 2 . Mothers of younger (4 - 14 weeks old, i.e. early postnatal group) and older (6 - 12 months old, i.e. midpostnatal group) children, representing HIV-exposed and unexposed children, were included to broadly represent PMTCT programme impact. A minimum target sample size of 120 mother-infant pairs was used for each of the four strata (i.e. an HIV-positive and an HIVnegative group of mothers in each of the two postnatal stages) per district. In each district, 24 medium to large facilities were randomly selected, and an average of five mothers were targeted per stratum in each facility. From the above postnatal sample, HIV-positive mothers were interviewed for assessing quality of VL care received. To measure TATs for VL results, a randomly selected equal sample of five PMTCT client records, from facility longitudinal registers at any point along the PMTCT cascade, was targeted per facility.

\section{Quality of VL care received}

Quality of VL care was defined as the explanation of VL results to a patient during result receipt as part of routine HIV counselling activities. We measured it from participants' recall of the meaning of their most recent VL result being explained to them (here referred to as 'expected' quality of VL care) or not explained to them (i.e. 'low' quality of VL care). Participants who reported not having collected their most recent VL result were included in the 'low-quality' group. In addition, we described participants' ability to recall the actual VL value. All participants who ever received a VL test during or after the most recent pregnancy were included.

\section{TAT of VL monitoring}

Three dates were extracted (from facility registers) from each randomly selected PMTCT client record to calculate TAT in days from the date of blood draw to the date on which the client received her VL results: the date of blood draw (which in all cases was the same as the date the sample was couriered to the laboratory), the date the facility received hard-copy VL results from the laboratory, and the date the client received results. The overall TAT to client for VL results was calculated as the date the client received results minus the date of blood draw. We were also interested in further understanding the total laboratory TAT (the date the facility received VL results from the laboratory minus the date the sample was couriered to the laboratory) and the clinical TAT (the date the client received results minus the date the facility received results from the laboratory).

\section{Statistical methods}

The study was designed to present results at district level. The quality of VL care was analysed using individual participant data, and all proportions and point estimates were weighted to account for sample size realisation within each stratum and the sampling frame of facilities within each district. A $\chi^{2}$ test was used to describe the quality of VL care. A logistic regression model was used to determine associations between quality of VL care received and the participant's sociodemographic and healthcare uptake characteristics, adjusted for postnatal period. Available participant sociodemographic information and healthcare uptake history were included together with socioeconomic status (SES) group. SES was calculated from household assets and source of income using principal component analyses and classified into high and low SES.

All three types of TATs for VL were presented as binary, 'short TAT' ( $\geq 80 \%$ of records with TAT $\leq 7$ days) and 'delayed TAT' $(<80 \%$ of records with TAT $\leq 7$ days). A $\chi^{2}$ test was used to describe TATs for VL between districts and Spearman's correlation to describe the relationship between total laboratory TAT and clinical TAT within each district. A logistic regression model was used to investigate the relationship between overall TAT to client and operational and infrastructural factors (i.e. methods used to receive VL results, use of any point-of-care (PoC) technology for VL care, and systems for tracing participants and monitoring retention in care). To determine whether the quality of VL care received by clients in a facility was related to the average overall TAT to clients observed at that facility, we calculated a logit-based risk difference in the quality of VL care received between facilities having short $\mathrm{v}$. those with delayed overall TAT to client. The risk difference analysis was adjusted for all participant factors showing a significant association with quality of VL care received, in any district.

All analyses were performed in Stata SE14 (StataCorp, USA), and a $5 \%(p<0.05)$ significance level was used.

\section{Ethical considerations and consent to participate}

Ethics approval for the study was granted by the South African Medical Research Council Ethics Committee in 2015 (ref. no. IDEC039-11/2015) and the Associate Director of Science, Center for Global Health at the US Centers for Disease Control and Prevention. All participants signed informed consent.

\section{Results}

\section{Sample summary}

We enrolled 853 HIV-positive mothers (totalling to $60 \%$ sample size realisation) from 132 facilities. Overall, 769 participants (90.2\%) reported ever having a VL test since the first antenatal care visit and were included in the analyses for quality of VL care. As expected, half $(50.7 \%)$ of the sample comprised the early postnatal group. Only $10.7 \%(n=82 / 769)$ of HIV-positive mothers could recall their actual VL. 
Ninety one percent $(n=120)$ of the facilities had at least $80 \%$ of reviewed client records with complete dates for measuring VL TATs ( $n=16 / 20$ in Rural NW, $n=17 / 21$ in Urban GP, $n=22 / 22$ in Urban KZN, $n=22 / 22$ in Rural/periurban MP, $n=23 / 23$ in Periurban EC and $n=20 / 24$ in Rural LP).

\section{Quality of VL care received}

The proportion of clients who received the expected quality of VL care was $>65 \%$ in all facilities (data not shown) and in each postnatal group (Fig. 1). The highest outcomes were observed in Rural/periurban MP $(81.6 \%$ and $85.2 \%$ for mid- and early postnatal groups, respectively). Rural NW and Urban GP followed closely (ranging between $77.5 \%$ and $82.4 \%$ ). There was no significant difference in proportions between postnatal groups, but proportions for the early postnatal groups were higher in most districts. The majority $(n=60 / 82)$ of clients who could recall their VL reported a VL of $>1000$ copies $/ \mathrm{ml}$, and only $4 / 82$ reported an undetectable VL.

The quality of VL care received was significantly associated with maternal age, education, unplanned pregnancy, timing of ART initiation and having experienced clinic visit barriers (i.e. hindrances to keeping clinic appointments such as lack of family support, transport issues and clinic waiting times (Table 1), in at least one of three districts (both urban metros and Rural LP) (Table 2). In Urban GP, the odds of receiving the expected quality of VL care was significantly reduced among postnatal mothers who had: (i) achieved primary-level education or less (adjusted odds ratio (aOR) 0.1; 95\% confidence interval (CI) $0.0-0.7 ; p=0.024$ ); (ii) an unplanned pregnancy (aOR $0.2 ; 95 \%$ CI 0.0 - 0.7; $p=0.020$ ); (iii) initiated ART after enrolling into antenatal care for the most recent pregnancy (i.e. recent ART initiation) (aOR 0.1; 95\% CI $0.0-0.7 ; p=0.020$ ); or (iv) experienced clinic visit barriers (aOR 0.1; 95\% CI 0.0 - 0.6; $p<0.001$ ), while mothers aged 25 - 34 years had a nearly 6 times odds of receiving the expected quality of VL care compared with older mothers (aOR 5.7; 95\% CI $1.1-29.5 ; p=0.037)$. In the second urban metro, Urban KZN, the odds of receiving the expected quality of care was significantly reduced only among postnatal mothers who experienced clinic visit barriers (aOR 0.2; $95 \%$ CI $0.1-0.9 ; p=0.039$ ). The odds of receiving the expected quality of $\mathrm{VL}$ care in Rural LP was significantly reduced among postnatal mothers who had: (i) primary-level education or less (aOR $0.1 ; 95 \%$ CI $0.0-0.7$; $p=0.024$ ); (ii) initiated ART after enrolling

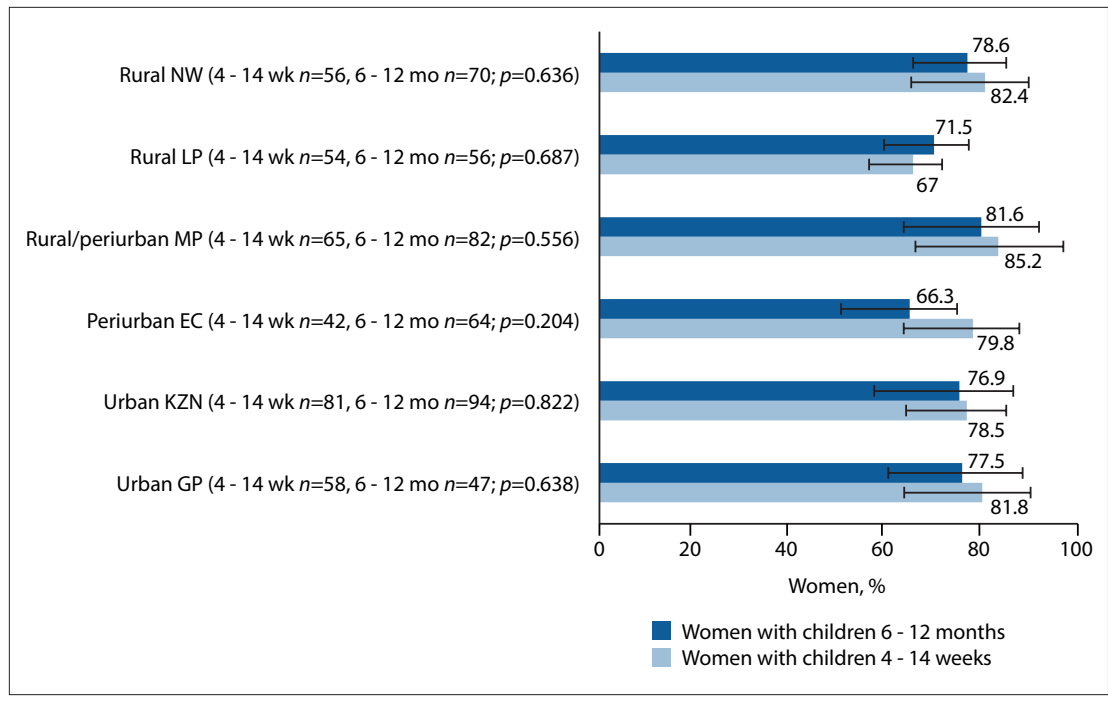

Fig. 1. Proportion of women with viral load results explained to them ( $\mathrm{N}=769$ women interviewed). The $\mathrm{p}$-values are from a $\chi^{2}$ test comparing proportions between postnatal groups. Horizontal lines to the right of the bars represent 95\% confidence intervals. (Rural NW = Bojanala District in North West Province; Rural LP = Greater Sekhukhune District in Limpopo Province; Rural/periurban MP = Ehlanzeni District in Mpumalanga Province; Periurban EC $=$ OR Tambo District in Eastern Cape Province; Urban KZN = eThekwini District in KwaZulu-Natal Province; Urban GP = Ekurhuleni District in Gauteng Province.)

Table 1. Factors reported to affect adherence to clinic appointments among clients who reported facing barriers to attending scheduled clinic visits $(N=519)$

\begin{tabular}{lll}
\hline Factor & $\boldsymbol{n}$ & Weighted \% out of 519 \\
\hline Waiting time at the clinic & 268 & 50.6 \\
Support from partner & 79 & 15.6 \\
Transport challenges & 72 & 13.9 \\
Support from family & 66 & 12.8 \\
Fear of being sick & 49 & 10.3 \\
Financial related & 56 & 10.1 \\
Stigma & 35 & 7.1 \\
Healthcare worker attitudes & 34 & 5.0 \\
Work or school attendance & 12 & 2.8 \\
Healthcare worker support & 13 & 2.8 \\
Household chores & 16 & 2.7 \\
No food & 7 & 2 \\
Peer support & 7 & 1.5 \\
No disclosure of HIV status & 8 & 1.1 \\
Disclosure of HIV status & 10 & 1
\end{tabular}

into antenatal care (aOR 0.2; 95\% CI 0.1 $0.8 ; p=0.024)$; or (iii) experienced clinic visit barriers (aOR 0.1 ; $95 \%$ CI $0.0-0.6$; $p=0.011$ ).

\section{TAT of VL monitoring}

The majority of facilities in all districts were supported by short total laboratory TAT, ranging from $50.0 \%$ in Rural LP to $86.4 \%$ in Rural/periurban MP (Fig. 2). Urban GP had $64.7 \%$ of facilities supported by short total laboratory TAT, and the remaining three districts had $72-75 \%$. The $\chi^{2} p$-values indicated uniformity in total laboratory TAT support across all districts. Clinical TAT varied widely between districts. Only two districts had at least half of their facilities achieving a short clinical TAT (Rural/ periurban MP with the highest at $63.6 \%$ and Rural NW with 50\%). Both urban metro districts had the lowest proportions of facilities achieving an average short clinical TAT (13.6\% of facilities in Urban KZN and zero facilities in Urban GP). Overall, TAT to client resembled clinical TAT, although only Rural/periurban MP had half of the facilities achieving the desired short overall TAT to client. Rural NW had $37.5 \%$ of facilities achieving a short overall TAT to client and 


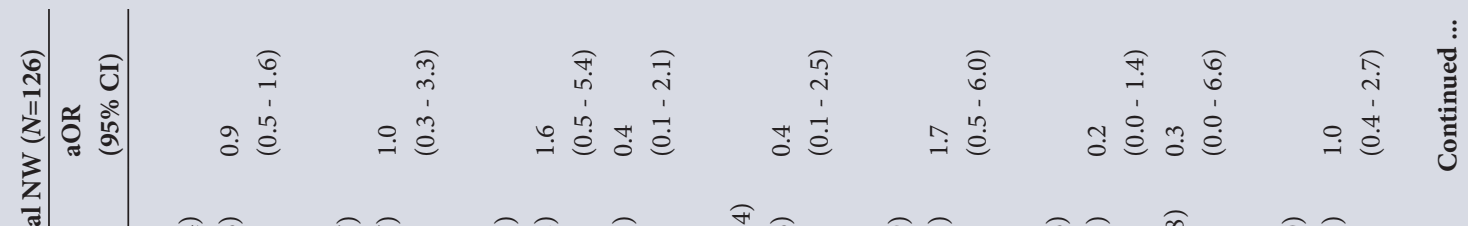

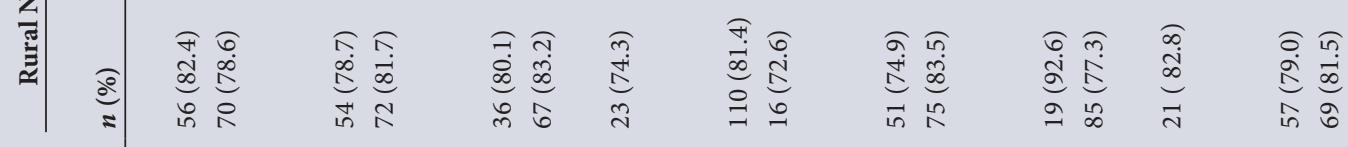

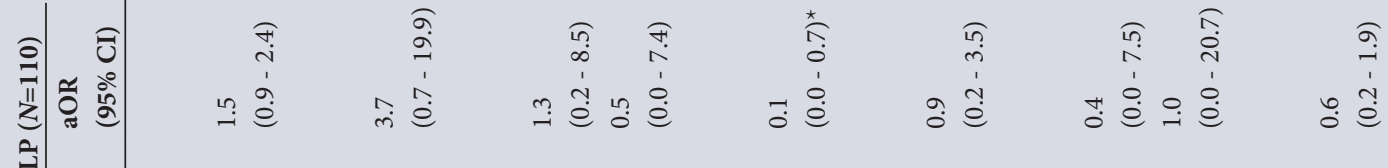

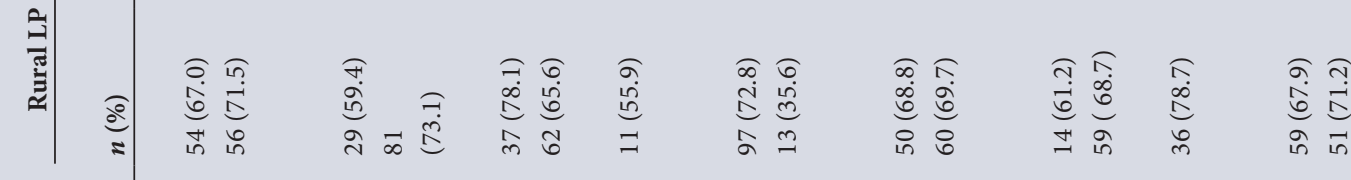

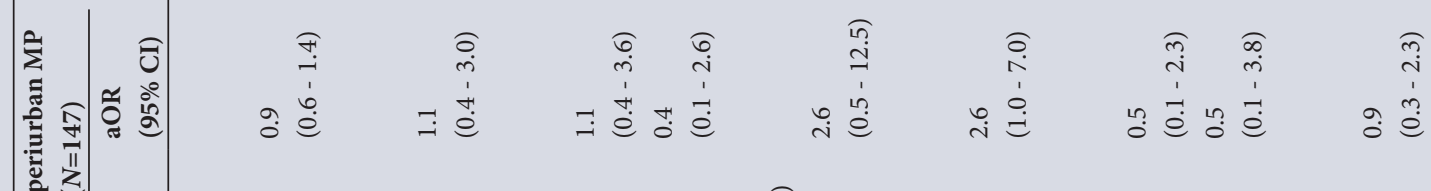

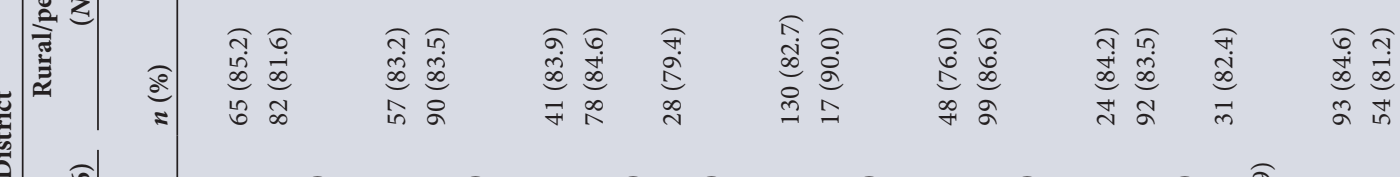

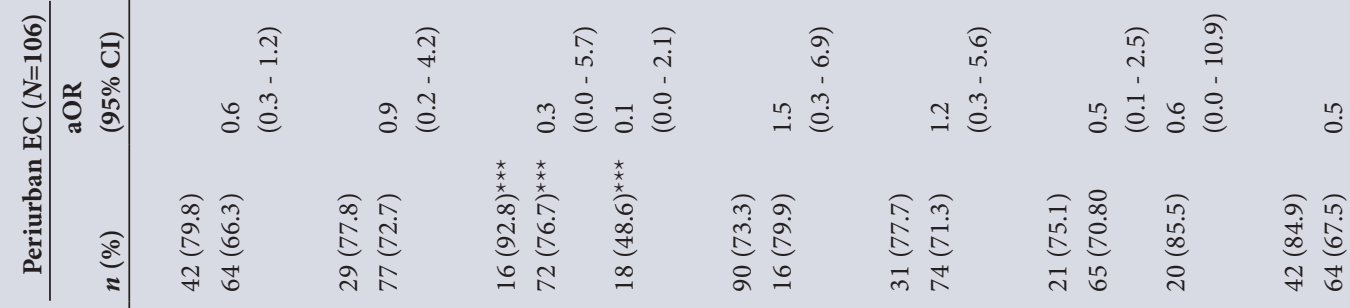

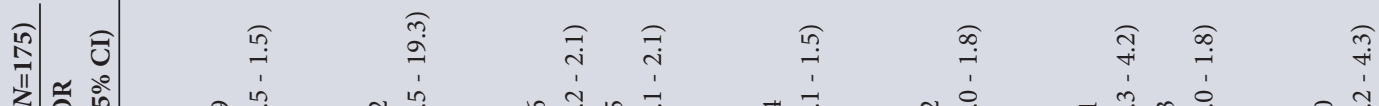
Z

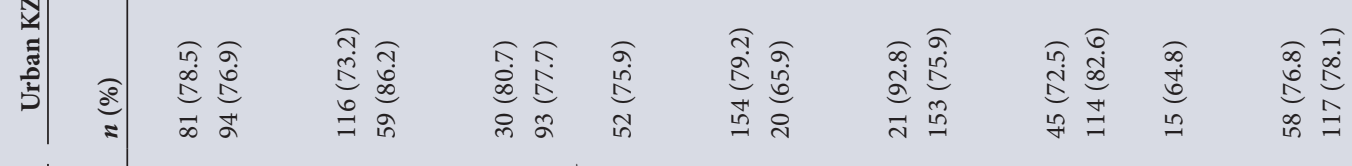

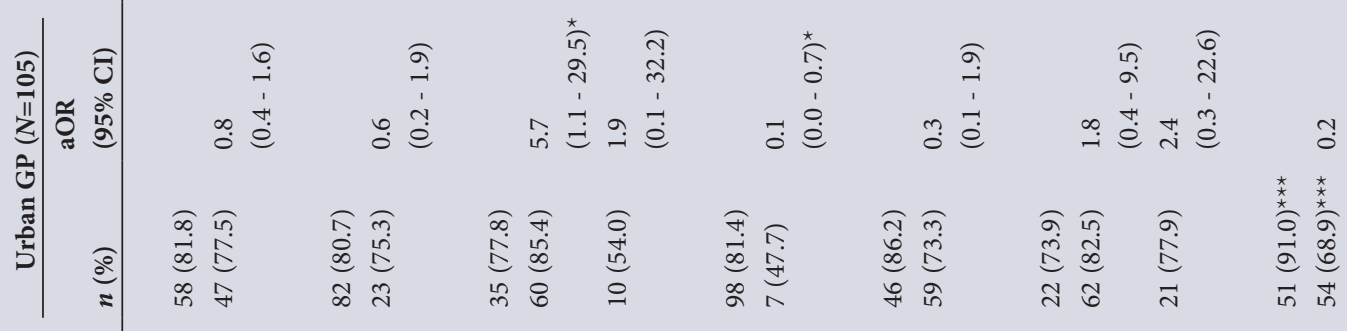


党

$\pi$

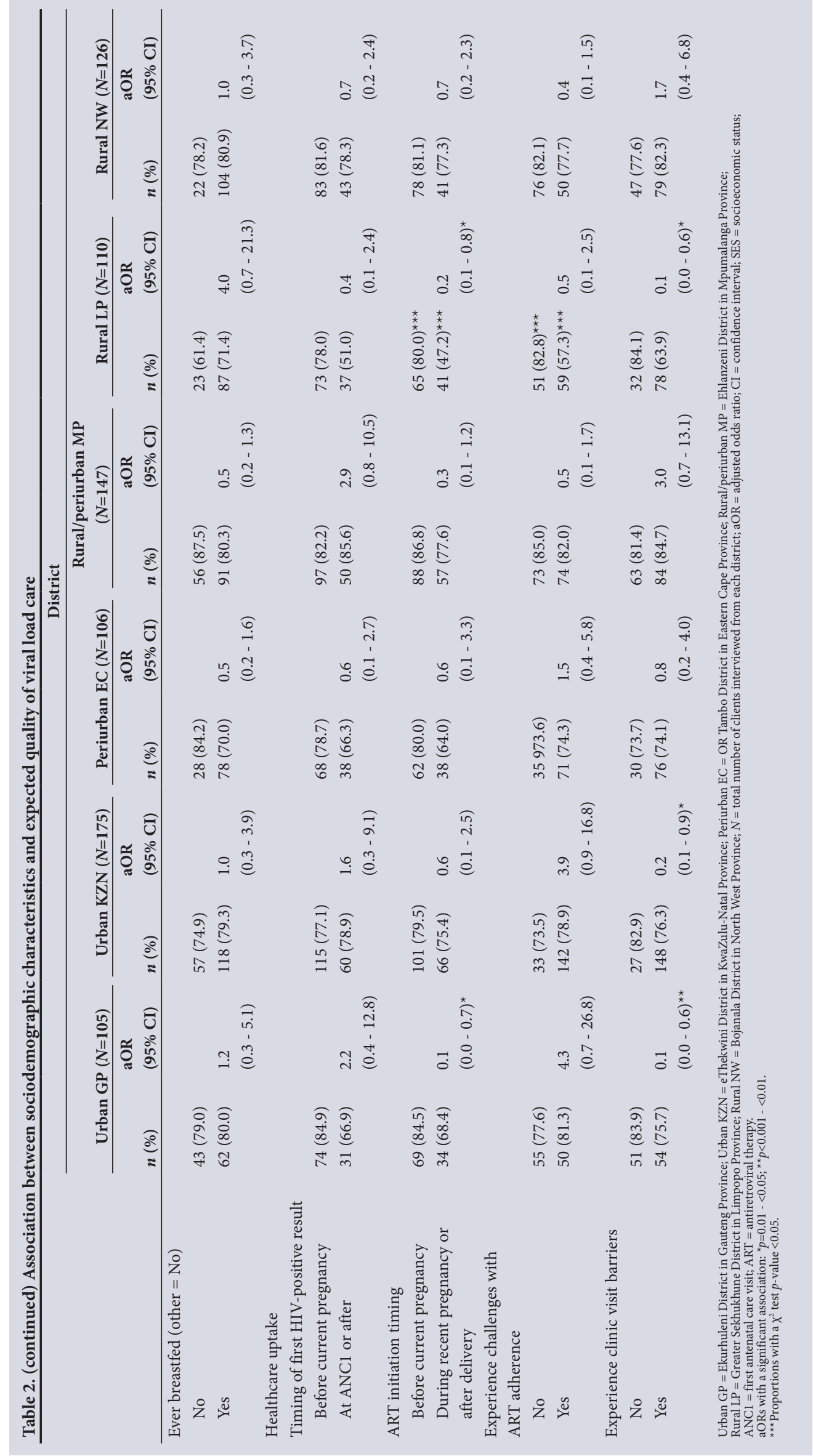




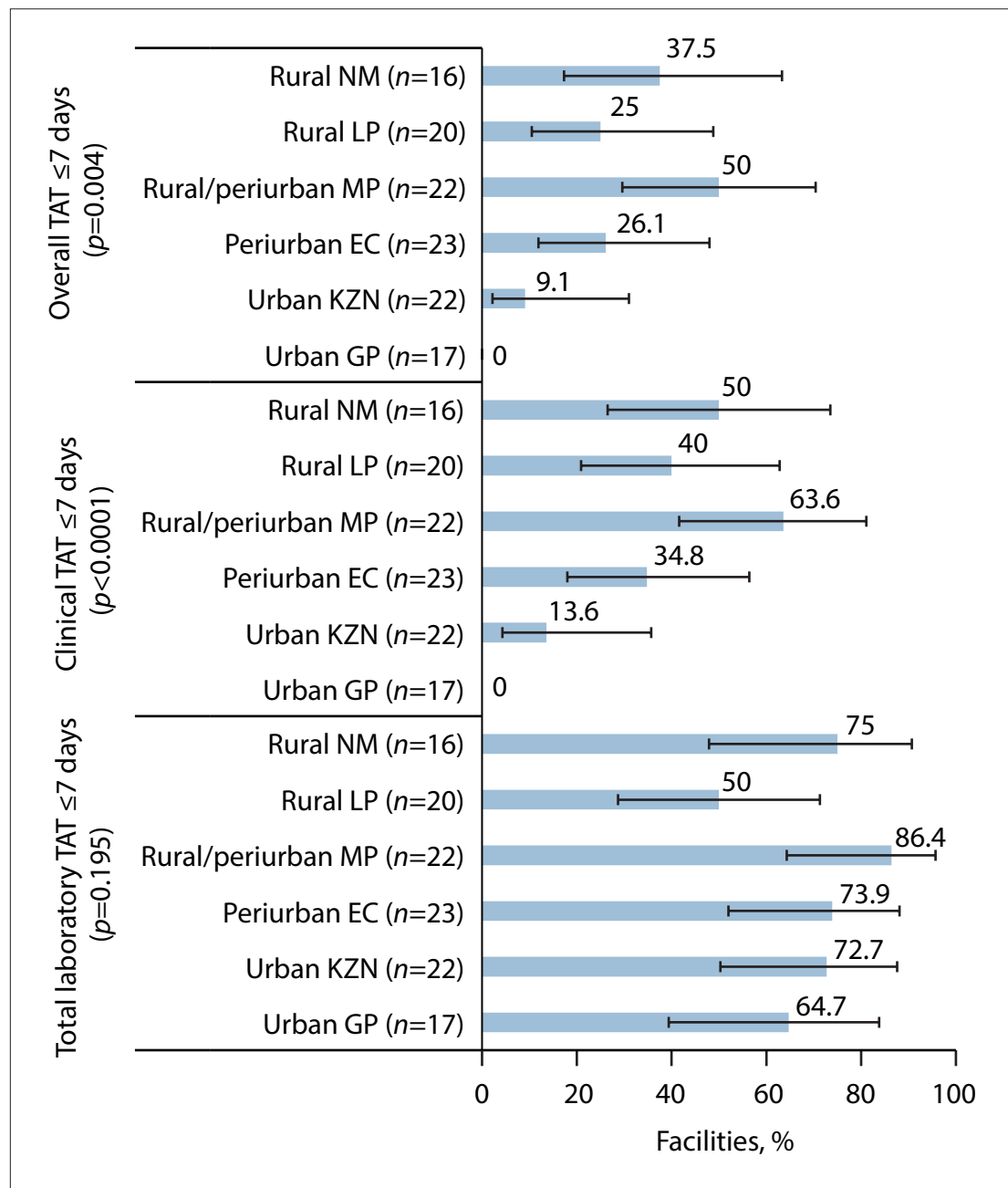

Fig. 2. Proportion of facilities with short TATs for viral load results. The $\mathrm{p}$-values are from a $\chi^{2}$ test. Horizontal lines to the right of the bars represent $95 \%$ confidence intervals. (TAT = turnaround time; Total laboratory $T A T=$ the time period including courier of blood sample to the laboratory, processing time in the laboratory and return of results to the facility; Clinical TAT = the time period results were kept in the facility after receipt from the laboratory and before client collection; Overall TAT $=$ the sum of clinical TAT and total laboratory TAT; Rural NW = Bojanala District in North West Province; Rural $L P=$ Greater Sekhukhune District in Limpopo Province; Rural/periurban MP = Ehlanzeni District in Mpumalanga Province; Periurban EC = OR Tambo District in Eastern Cape Province; Urban KZN = eThekwini District in KwaZulu-Natal Province; Urban GP = Ekurhuleni District in Gauteng Province; $\mathrm{n}=$ number of healthcare facilities surveyed.)

Rural LP had 25.0\%. No facility achieved this in Urban GP, and only $9.1 \%$ did so in the other urban metro.

Clinical TAT and total laboratory TAT were unrelated (all $p$-values $>0.05$ for Spearman's correlation, see Supplementary File 1, available at http://samj.org.za/public/ sup/15496.pdf).

No facility-level infrastructural and operational factors evaluated were significantly associated with overall TAT to client (Table 3).

\section{Relationship between quality and TAT of VL monitoring}

The risk difference for quality of VL care received between short overall TAT to client v. delayed TAT at facility level was evaluated. The quality of VL care received by clients was not significantly associated with the respective facility's average overall TAT to client, with adjustment for individual client characteristics significantly associated with quality of care at any district (Table 4). However, short overall TAT to client was significantly associated with the risk of unplanned pregnancy and not having experienced clinic visit barriers in Rural LP and Periurban EC, respectively. In Rural LP facilities, short overall TAT to client was significantly associated with a 0.28 percentage point (adjusted risk difference (ARD) $0.28 \%$; 95\% CI $0.07-0.49 ; p=0.015$ ) increased risk of having had an unplanned pregnancy. In Periurban EC facilities, short overall TAT to client was significantly associated with a 0.41 percentage point (ARD 0.41\%; 95\% CI $-0.63-0.21 ; p<0.001$ ) reduced risk of having experienced clinic visit barriers.

\section{Discussion}

Evaluation of VL monitoring service delivery within the PMTCT cascade is needed for understanding the risk of MTCT and barriers to achieving the third 90 of the UNAIDS 90-90-90 goals. To contribute to fulfilling this need, we evaluated: ( $i$ ) the quality of VL care received by PMTCT clients using routine healthcare facilities; and (ii) average TATs for return of VL results to PMTCT clients achieved by healthcare facilities, in six SA districts with a high maternal and infant HIV burden. We observed, according to client recall, that over two-thirds of them received the expected quality of care. However, $<40 \%$ of facilities in five districts $(50 \%$ in the sixth district) achieved optimal TATs for returning VL results to clients. The expected quality of VL care was significantly associated with maternal age, education, unplanned pregnancy, timing of ART initiation and experiencing clinic visit barriers. None of the infrastructural and operational factors linked to VL monitoring systems were significantly associated with average facility TATs. However, we observed that total laboratory TAT was consistent between districts, while clinical TAT varied significantly and influenced the significant variation observed for the overall TAT for VL. Overall facility TAT was not related to the quality of VL care received in the facilities, but was associated with unplanned pregnancy and experiencing clinic visit barriers.

Factors significantly associated with quality of VL care were observed in both urban metros and one rural district. Clinic visit barriers, low education, recent ART initiation and unplanned pregnancy were associated with not receiving the expected quality of care, the latter factor being common across all three districts. Clinic visit barriers cited by clients included common problems in similar low- to middle-income country settings, i.e. waiting times in the clinic, family/partner support, transport and financial-related challenges (Table 1). ${ }^{[6-8]} \mathrm{A}$ positive note in one periurban district was that facilities achieving overall short TATs were significantly less likely to have clients reporting clinic visit barriers.

The association of low educational qualification with poor quality of HIV care/ uptake is another persisting problem in SA. ${ }^{[9]}$ Unlike those with higher education, these 
Table 3. Association between overall TAT to client and infrastructural factors

\begin{tabular}{|c|c|c|c|c|c|c|}
\hline & $\begin{array}{l}\text { Total } \\
\text { facilities, } N\end{array}$ & $\begin{array}{l}\text { Facilities with } \\
\text { overall client } \\
\text { TAT } \leq 7 \text { days, } \%\end{array}$ & $\begin{array}{l}\text { Unadjusted } \\
\text { OR }(95 \% \mathrm{CI})\end{array}$ & $p$-value & aOR $(95 \% \mathrm{CI})$ & $p$-value \\
\hline \multicolumn{7}{|l|}{ Main method of receiving VL results } \\
\hline $\begin{array}{l}\text { SMS (text message) printer/email/online access, } \\
\text { such as Tier }\end{array}$ & 15 & 13.3 & Ref. & & & \\
\hline NHLS courier & 105 & 26.7 & $2.3(0.5-11.1)$ & 0.277 & $1.8(0.3-10.6)$ & 0.533 \\
\hline \multicolumn{7}{|l|}{ Use of PoCT for VL } \\
\hline No & 69 & 26.1 & Ref. & & & \\
\hline Yes & 51 & 23.5 & $0.9(0.4-2.0)$ & 0.749 & $1.0(0.4-2.5)$ & 0.972 \\
\hline \multicolumn{7}{|l|}{ Trace clients with high-risk HIV results } \\
\hline Not done & 9 & 33.3 & Ref. & & & \\
\hline Phone call only & 57 & 31.6 & $0.9(0.2-4.1)$ & 0.916 & $0.6(0.1-3.7)$ & 0.555 \\
\hline Counsellor/DOTs only & 16 & 18.8 & $0.5(0.1-3.0)$ & 0.418 & $0.6(0.1-5.4)$ & 0.655 \\
\hline Using phone call/counsellors/DOTs & 38 & 15.8 & $0.4(0.1-1.9)$ & 0.240 & $0.4(0.1-2.6)$ & 0.312 \\
\hline \multicolumn{7}{|l|}{ Has system to monitor retention in care for 1 year } \\
\hline No & 16 & 25.0 & Ref. & & & \\
\hline Yes & 104 & 25.0 & $1.0(0.3-3.4)$ & 1.000 & $1.3(0.4-5.1)$ & 0.665 \\
\hline \multicolumn{7}{|l|}{ District } \\
\hline Periurban EC & 23 & 26.1 & Ref. & & & \\
\hline Urban GP & 17 & 0.0 & 1 & & & \\
\hline Urban KZN & 22 & 9.1 & $0.3(0.1-1.6)$ & 0.152 & $0.4(0.1-2.6)$ & 0.332 \\
\hline Rural/periurban MP & 22 & 50.0 & $2.8(0.8-9.9)$ & 0.103 & $2.9(0.7-11.4)$ & 0.137 \\
\hline Rural LP & 20 & 25.0 & $0.9(0.2-3.7)$ & 0.935 & $1.1(0.2-4.7)$ & 0.939 \\
\hline Rural NW & 16 & 37.5 & $1.7(0.4-6.7)$ & 0.449 & $2.0(0.4-9.1)$ & 0.367 \\
\hline
\end{tabular}

clients are likely to feel disempowered to engage proactively with healthcare workers, so HIV counselling needs to be a universal standard procedure at every HIV-related healthcare contact. It is unclear why only a tenth of participants, most of whom had very high VLs, could recall their VL, even though more than half of the participants had reached high school. The reason is probably a combination of ill health-driven (or high VL) need to memorise one's VL, healthcare worker commitment, more recent collection of the VL result, service delivery culture or lack of quality improvement initiatives. ${ }^{[10-12]}$

Recent ART initiation significantly reduced the odds of receiving expected care in two districts. The Option B+ policy follows a test-andtreat strategy, so this group is likely to have been diagnosed with HIV recently. Related findings of poor healthcare outcomes among PMTCT clients with recent ART initiation have been reported across subSaharan Africa, highlighting a need for early linkage to complementary peer or other forms of community-based support. ${ }^{[8,13-16]}$ The home nurse programme in Tanzania and the postnatal peer-support clubs model currently used in some parts of SA are good examples for consideration nationwide. ${ }^{[17,18]}$

The association between unplanned pregnancy and undesirable HIV care outcomes is also not new in these settings, and interventions to reverse this chronic problem are overdue. ${ }^{[1,20]}$ The unexpected positive association with short TAT in Rural LP facilities is worth noting, however. The majority (70\%) of women reporting unplanned pregnancy in this district were already on ART before enrolling into antenatal care. The cross-sectional study design is limiting to understand temporality in this outcome. We could hypothesise that tracing high-risk clients identified during pregnancy, to reduce vertical HIV transmission risk, is prioritised in this district.
The WHO-recommended target for eliminating vertical HIV transmission to $\leq 50$ new HIV infections per 100000 live births has not been met in SA, and maintaining $>90 \% \mathrm{VL}$ suppression in HIV-positive mothers would reduce the risk of MTCT. ${ }^{[21]}$ In order to meet these goals, the previous national PMTCT guidelines expected VL-informed HIV care to be issued within 2 weeks, while the recently revised guidelines (2019) recommend that the healthcare worker check VL results and call the client within a week from the time of blood draw. ${ }^{[4,5]}$ The TATs we observed here question whether it is feasible to maintain viral suppression in $>90 \%$ of PMTCT clients across the entire period of pregnancy and breastfeeding. Inefficient TATs in HIV care are still a common problem in other African settings. ${ }^{[11,12,22-27]}$ Although fewer facilities managed to achieve desirable TATs in the present study, the unbiased and shorter laboratory TATs are a promising outcome for SA. However, the fact that a largely rural district can achieve better overall outcomes than urban districts, and that merely having PoC technologies does not guarantee overall optimal TATs, reiterates the complexity of achieving optimal VL monitoring processes. ${ }^{[11,28]}$ While the urban metro, with delayed clinical and overall TATs in all its facilities, is concerning, the rural/periurban district presenting better TAT outcomes indicates that efficient systems are possible. Ongoing quality improvement initiatives such as that implemented in Uganda have potential to improve service delivery processes and should be adopted widely. ${ }^{[10,29]}$ SA is currently piloting a digitised system called eLABs to track and improve VL monitoring systems and TATs. ${ }^{[30]}$

\section{Study limitations}

The sample size for the assessment of TATs was only powered to give average performance at facility level, and a patient-level assessment of whether women are being monitored for VL according to guidelines 


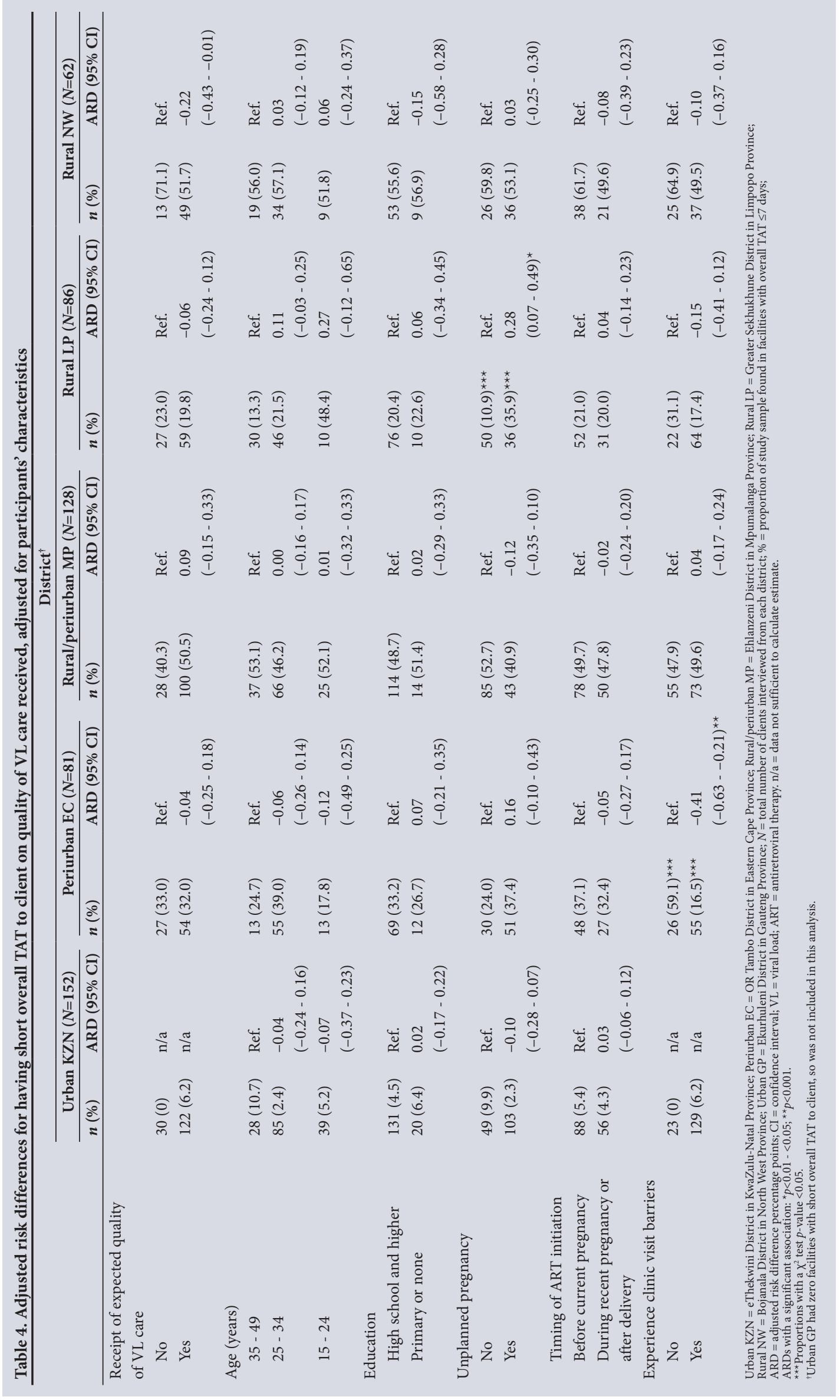


is needed. Although hard-copy records are the main source for TAT information, we did not consider that sometimes facilities use electronic tracking systems concurrently to expedite patient care. The time since the recent VL test was not considered and could have introduced recall bias. The contribution of service provider factors to the quality of VL care was not assessed. The study was designed to report district-level outcomes for individual patient data, so pooled analyses could not be done to benefit from the power of a larger sample size. However, the study was able to show that service delivery performance differs between districts, mirroring the heterogenous prevalence of antenatal HIV and MTCT, and further emphasising the need for interventions tailored to address challenges at the subregional contexts. ${ }^{[3]}$ The cross-sectional nature of the study design limits our understanding of temporality, especially for associations between short TAT and unplanned pregnancy.

\section{Conclusions}

This study has provided preliminary findings that will help to inform improved programme interventions to address some bottlenecks to monitor the third 90 of the UNAIDS 90-90-90 goals within the PMTCT cascade. The total laboratory TATs in SA are promising and reflect the country's efforts to leverage laboratory services to all settings. There is clearly a challenge in getting VL results back to clients from the facilities, and this appears to be more problematic in urban settings and contributed to by client-related barriers to clinic attendance. Delayed return of results to clients is particularly concerning during PMTCT, as rapid viral suppression is required to reduce the risk of transmission. The increasing use of mobile technology such as the MomConnect mobile service for promotional healthcare messaging provides an opportunity to explore new avenues to encourage women to collect their results timeously. ${ }^{[31]}$ The current roll-out of the eLABs system for digitised tracking and improving service delivery efficiency for VL monitoring could improve outputs. ${ }^{[30]}$ The quality of VL care was above average, but quality improvement initiatives need to continue. Periodic evaluation of the efficiency and quality of VL monitoring processes in countries that have not met the targets for eliminating MTCT is non-negotiable.

\section{Declaration. None.}

Acknowledgements. We thank Prof. Carl Lombard for providing overall guidance on the sample size design and statistical approach for the overall primary study, and other members of the Health Systems Research Unit PMTCT team who were involved in design, conduct and data management of the primary study - Nobuntu Makhari, Natasha Titus, Trisha Ramraj, Lucille Heyns and Vincent Maduna.

Author contributions. NKN: conceptualised manuscript objectives, carried out analyses and wrote the first draft of the manuscript; TM: reviewed the literature for global and national PMTCT guidelines for VL monitoring; AEG, DFN, GGS, VM, WC: critically reviewed the first draft of the manuscript and contributed to subsequent versions. NKN, DFN, WC, VM, AG were part of the primary study team. All authors reviewed and agreed on the final version of the manuscript.

Funding. This project has been supported by the President's Emergency Plan for AIDS Relief (PEPFAR) through the Centers for Disease Control and Prevention (CDC) (under the terms of the Collaboration Agreement Grant number 5U2GGH001150). The publication of this manuscript was funded by the South African Medical Research Council. The findings and conclusions in this report are those of the authors and do not necessarily represent the official position of the funding agencies.

Conflicts of interest. None.
1. Myer L, Phillips TK, McIntyre JA, et al. HIV viraemia and mother-to-child transmission risk after antiretroviral therapy initiation in pregnancy in Cape Town, South Africa. HIV Med 2017;18(2):80-88. https://doi.org/10.1111/hiv.12397

2. Joint United Nations Programme on HIV/AIDS (UNAIDS). 90-90-90: An ambitious treatment target to help end the AIDS epidemic. October 2014. http://files.unaids.org/en/media/unaids/contentassets to help end the AspS epidemic. October 2014. http://files.unaids.org/en/media/

3. Woldesenbet SA, Kufa T, Lombard C, et al. The 2017 National Antenatal Sentinel HIV Survey, South Africa. National Department of Health, South Africa, 2019. https://www.nicd.ac.za/wp-content/ Africa. National Department of Health, South Africa, 2019. https://www.nicd.ac
uploads/2019/07/Antenatal_survey-report_24July19.pdf (accessed 26 November 2019).

National Department of Health, South Africa. Guideline for the prevention of mother to child

National Department of Health, South Africa. Guideline for the prevention of mother to child transmission of communicable infections. October 2019. https://www.knowledgehub.org.za/elibrary guideline-prevention-moter

National Department of Health, South Africa. National consolidated guidelines for the prevention of mother-to-child transmission of HIV (PMTCT) and the management of HIV in children, adolescents and adults. 2 July 2015. https://health-e.org.za/2015/07/02/guidelines-national-consolidated-guideline for-pmtct-and-the-management-of-hiv-in-children-adolescents-and-adults/ (accessed 17 May 2019).

6. Clouse K, Schwartz S, van Rie A, et al. 'What they wanted was to give birth; nothing else': Barriers to retention in option B+ HIV care among postpartum women in South Africa. J Acquir Immune Defic Syndr 2014;67(1):e12-18. https://doi.org/10.1097/QAI.0000000000000263

Yehia BR, Stewart L, Momplaisir F, et al. Barriers and facilitators to patient retention in HIV care. BMC Infect Dis 2015;15:246. https://doi.org/10.1186/s12879-015-0990-0

8. Muchedzi A, Chandisarewa W, Keatinge J, et al. Factors associated with access to HIV care and treatment in a prevention of mother to child transmission programme in urban Zimbabwe. J Int AIDS Soc 2010;13(1):38. https://doi.org/10.1186/1758-2652-13-38

9. Woldesenbet S, Jackson D, Lombard C, et al. Missed opportunities along the prevention of mother-tochild transmission services cascade in South Africa: Uptake, determinants, and attributable risk (the SAPMTCTE). PLoS ONE 2015;10(7):e0132425. https:///doi.org/10.1371/journal.pone.0132425

10. Ochola E, Aloyo J, Rahimzai M. Increasing viral load monitoring of people living with HIV on AR' in Northern Uganda in line with the 90-90-90 global targets. United States Agency for Internationa Development (USAID), United States President's Emergency Plan for AIDS Relief (PEPFAR), June 2016 https://www.urc-chs.com/sites/default/files/improving_vl_testing_in_northern_uganda_june2016 usltr_ada.pdf (accessed 10 March 2020).

11. Shiferaw MB, Yismaw G. Magnitude of delayed turnaround time of laboratory results in Amhara Public Health Institute, Bahir Dar, Ethiopia. BMC Health Serv Res 2019;19(1):240. https://doi.org/10.1186/ s12913-019-4077-2

2. Saito S, Duong YT, Metz M, et al. Returning HIV-1 viral load results to participant-selected health facilities in national population-based HIV impact assessment (PHIA) household surveys in three sub-Sahara African countries, 2015 to 2016. PLoS ONE 2017;20(S7):e25004. https://doi.org/10.1002/jia2.25004

3. Clouse K, Hanrahan CF, Bassett J, et al. Impact of systematic HIV testing on case finding and retention in care at a primary care clinic in South Africa. Trop Med Int Health 2014;19(12):1411-1419. https://doi. org $/ 10.1111 /$ tmi.12387

4. Tenthani $\mathrm{L}$, Haas $\mathrm{AD}$, Tweya $\mathrm{H}$, et al. Retention in care under universal antiretroviral therapy for HIV-infected pregnant and breastfeeding women ('Option B+') in Malawi. AIDS (London, England) 2014;28(4):589-598. https://doi.org/10.1097/QAD.0000000000000143

15. Phiri S, Tweya $\mathrm{H}$, van Lettow $M$, et al. Impact of facility- and community-based peer support models on maternal uptake and retention in Malawis Option B+ HIV prevention of mother-to-child transmission program: A 3-arm cluster randomised controlled trial (PURE Malawi). J Acquir Immune Defic Syndr

16. Technau K-G, Kalk E, Coovadia A, et al. Timing of maternal HIV testing and uptake of prevention of mother-to-child transmission interventions among women and their infected infants in Johannesburg. South Africa. J Acquired Immune Deficiency Syndr 2014;65(5):e170-e178. https://doi.org/10.1097/ QAI.0000000000000068

17. Manumbu S, Smart LR, Mwale A, et al. Shortening turnaround times for newborn HIV testing in rural Tanzania: A report from the field. PLoS Med 2015;12(11):e1001897. https://doi.org/10.1371/journal. pmed.1001897

18. Duvivier H, Decroo T, Nelson A, et al. Knowledge transmission, peer support, behaviour change and satisfaction in post natal clubs in Khayelitsha, South Africa: A qualitative study. Reprod Health 2020;17(1):107. https://doi.org/10.1186/s12978-020-00957-0

19. Adeniyi OV, Ajayi AI, Moyaki MG, et al. High rate of unplanned pregnancy in the context of integrated Adeniyi OV, Ajayi AI, Moyaki MG, et al. High rate of unplanned pregnancy in the context of integrated
family planning and HIV care services in South Africa. BMC Health Serv Res 2018:18(1):140. https://doi. family planning and HIV care ser
org/10.1186/s12913-018-2942-z

20. Iyun V, Brittain K, Phillips TK, et al. Prevalence and determinants of unplanned pregnancy in HIVpositive and HIV-negative pregnant women in Cape Town, South Africa: A cross-sectional study. BMI
punt positive and HIV-negative pregnant women in Cape Town, South Afric:

Open 2018;8(4):e019979. https://doi.org/10.1136/bmjopen-2017-019979 World Health Organization. Global guidance on processes and criteria for validation: Elimination of
mother-to-child transmission of HIV and syphilis. 2nd ed. Geneva: WHO, 2017. https://apps.who.int/iris/ mother-to-child transmission of HIV and syphilis. 2nd ed. Geneva: WHO, 2017. https:/

22. Glass TR, Motaboli L, Nsakala B, et al. The viral load monitoring cascade in a resource-limited setting.

Glass TR, Motaboli L, Nsakala B, et al. The viral load monitoring cascade in a resource-limited setting:
A prospective multicentre cohort study after introduction of routine viral load monitoring in rural A prospective multicentre cohort study after introduction of routine viral load mon
Lesotho. PLoS ONE 2019;14(8):e0220337. https://doi.org/10.1371/journal.pone.0220337

23. Roberts T, Cohn J, Bonner K, Hargreaves S. Scale-up of routine viral load testing in resource-poo settings: Current and future implementation challenges. Clin Infect Dis 2016;62(8):1043-1048. https:/ doi.org/10.1093/cid/ciw001

24. Gill M, Hoffman HJ, Mokone M, et al. Assessing very early infant diagnosis turnaround times: Finding from a birth testing pilot in Lesotho. AIDS Res Treat 2017:2572594. https://doi.org/10.1155/2017/2572594 5. Kroidl A, Burger T, Urio A, et al. High turnaround times and low viral resuppression rates after reinforced adherence counselling following a confirmed virological failure diagnostic algorithm in HIV-infected patients on first-line antiretroviral therapy from Tanzania. Trop Med Int Health 2020;25(5):579-589. https://doi.org/10.1111/tmi.13373

6. Mwau M, Syeunda CA, Adhiambo M, et al. Scale-up of Kenyass national HIV viral load program: Finding and lessons learned. PLoS ONE 2018;13(1):e0190659. https://doi.org/10.1371/journal.pone.0190659

27. Nyakura J, Shewade HD, Ade S, et al. Viral load testing among women on 'Option B+' in Mazowe, Zimbabwe How well are we doing? PLoS ONE 2019;14(12):e0225476. https://doi.org/10.1371/journal.pone.0225476

28. Deo S, Crea L, Quevedo J, et al. Implementation and operational research: Expedited results deliver systems using GPRS technology significantly reduce early infant diagnosis test turnaround times. J Acquir Immune Defic Syndr 2015;70(1):el-e4. https://doi.org/10.2139/ssrn.2510213

29. Tumwikirize J, Nakanwagi F, Kisamba H, Karamagi E, Rahimazi M. Improving viral load monitoring at health facilities providing HIV care: Tested changes and guidance from Uganda. Change Package. Published by the USAID ASSIST Project. Bethesda, MD: University Research Co., LLC (URC). 2017. https://pdf.usaid.gov/pdf_docs/PA00TC5G.pdf (accessed 25 May 2020).

30. Chugudu K, Kirsten T, Gorogodo B, Stevens W. Demonstration of usability of a digital intervention (DHI) for strengthening the clinic: Lab interface for HIV viral load testing in a South African clinical setting (eLABs). Presented at the 9th South African AIDS Conference, Durban, South Africa, 12 June 2019. http://www.saaids.co.za/.cm4all/uproc.php/0/Hall\%203/Mr\%20Kumbirai\%20Chigudu pdf?cdp=a\&_=16bdab03348 (accessed 2 October 2020).

31. Peter J, Barron P, Pillay Y. Using mobile technology to improve maternal, child and youth health an treatment of HIV patients. S Afr Med J 2016;106(1):3-4. https://doi.org/10.7196/SAMJ.2016.v106i1.10209 